\title{
Operative Behandlung von Wirbelfrakturen - ventrale Instrumentierung
}

\author{
Franz Holz, Stefan Matschke, Andreas Wentzensen
}

\section{Zusammenfassung}

Bei den häufig langfristig nicht zufriedenstellenden Ergebnissen rein dorsaler Operationsverfahren zur Behandlung instabiler Frakturen der Brustund Lendenwirbelsäule findet z.Z. eine Trendwende hin zu kombinierten OP-Techniken im Sinne von dorsoventralen Instrumentierungen und einer ventralen Fusionstechnik statt. Neue winkelstabile Implantate sowie die
Entwicklung von minimal-invasiven Verfahren verringern die Zugangsmorbidität deutlich, gleichzeitig wird durch Anwendung dieser OP-Technik der biomechanischen Situation der Wirbelsäule Rechnung getragen mit Wiederherstellung der lasttragenden vorderen Säule (Load sharing-System). Bei erfreulichen klinischen Ergebnissen fehlen bisher mittelfristige Ergebnisse bezüglich der Wirbelkörperersatzimplantate.

\section{Einleitung}

Unter funktionell anatomischen Gesichtspunkten bildet die Wirbelsäule eine komplexe Konstruktion einzelner Bewegungssegmente. Das Behandlungskonzept von Verletzungen der Wirbelsäule kann sich daher nicht allein auf die knöchernen Schäden an der Wirbelsäule beschränken. In die Behandlungsüberlegungen müssen deshalb die knöchernen Strukturen, die Intervertebralgelenke mit Gelenkkapsel, die Bandscheiben, die ligamentären Verbindungen und stabilisierenden Muskeln einbezogen werden.

Die anfängliche Euphorie bei der operativen Behandlung von Wirbelkörperfrakturen der Brust- und Lendenwirbelsäule, unabhängig von der Frakturklassifikation, allein durch pedikulär verankerte winkelstabile Platten- und Stabsysteme, konnte durch die mittelfristigen Ergebnisse nicht gestützt werden.

Korrekturverluste bis zum Erreichen der primär unfallbedingten Fehlstellungen traten auf.

OP-JOURNAL 2001; 17: 162-171

(c) Georg Thieme Verlag Stuttgart · New York
Auch die Einführung der transpedikulären Spongiosaauffüllung nach Ausräumen der Bandscheibe von dorsal und Ausfüllung des Inter- und Intravertebralraumes mit Spongiosa führte letztendlich häufig nicht zu der gewünschten ventralen knöchernen Durchbauung der zu fusionierenden Segmente. Teilweise wurde die transpedikuläre Spongiosa resorbiert, in vielen Fällen war die Vitalität des transplantierten Knochens fraglich, Korrekturverluste waren vorprogrammiert $[6,12]$.

Konsequenter Weise wurden, entsprechend biomechanischer Bedürfnisse, zunehmend direkte ventrale Fusionsverfahren eingeführt und eingesetzt.

\section{Biomechanik}

Auf die Wirbelsäule wirken Kompressionskräfte, Biegemomente, Rotationskräfte und Scherkräfte ein. Wegen des vor der Wirbelsäule liegenden Körperschwerpunktes bildet der knöcherne, ligamentäre und muskuläre Aufbau der Wirbelsäule ein System, welches in den ventralen knöchernen Abschnitten bei aufrechter Körperposition für eine axiale Belastung und in den dorsalen ligamentären Abschnitten für eine Zugspannung ausgelegt ist. Die biomechanischen Verhältnisse sind mit einem Kranmodell ver- gleichbar (Abb.1). Aus diesem Krankonstruktionsprinzip kann bildhaft abgeleitet werden, dass ventral die Wirbelsäule auf Druck belastet wird und in der dorsalen Gliederkette zur Balancierung in aufrechter Haltung Zugkräfte auftreten [11]. Dies bedeutet letztendlich, dass die dorsale Zuggurtung nur funktionieren kann, wenn die Integrität der vorderen Säulenkonstruktion intakt ist. Die vordere Säule muss einer Druckbelastung entgegen wirken, wo hingegen die hintere Gelenkkettenkonstruktion einer Zugbelastung ausgesetzt ist. Daneben kommt den diskalen und ligamentären Strukturen noch eine wesentliche Bedeutung bei der Kompensation von Rotation und Scherkräften zu.

Aus diesen Überlegungen ergibt sich, dass durch die dorsale Reposition mit Aufrichtung des verletzten Segmentes eine Verstärkung des ventralen Defektes und damit der Instabilität entsteht. Somit ist die Rekonstruktion der Tragfähigkeit der vorderen Säule für eine erfolgreiche operative Behandlung von Wirbelfrakturen erforderlich. Die Ausheilung einer Verletzung ist abhängig von der Ausheilung der biologisch zerstörten Gewebestruktur, die im Bereich des Wirbelkörpers $70-80 \%$ der Kraft überträgt. Für die Wirbelsäule bedeutet dies in der Regel das Erzielen einer knöchernen Fusion zur Restabilisierung und Herstellung der normalen anatomischen Form. Selten ist die Funktion eines verletzten Wirbelsäulensegmentes zu erhalten (z.B. Dens-Verschraubung).

Beachtet man die Wiederherstellung der ventralen Säule nicht, so führt das Einbringen eines dorsalen Distraktionsinstrumentariums zu einer Umkehrung des Load sharing-Prinzips. Es kommt dabei zu einer umgekehrten Krafteinleitung, d.h. ein Großteil der in die Wirbelsäule eingeleiteten Kraft läuft dorsal über das Instrumentarium. Matthis und Biedermann [8] konnten nachweisen, dass bei einer solchen Konstruktion 90\% der 


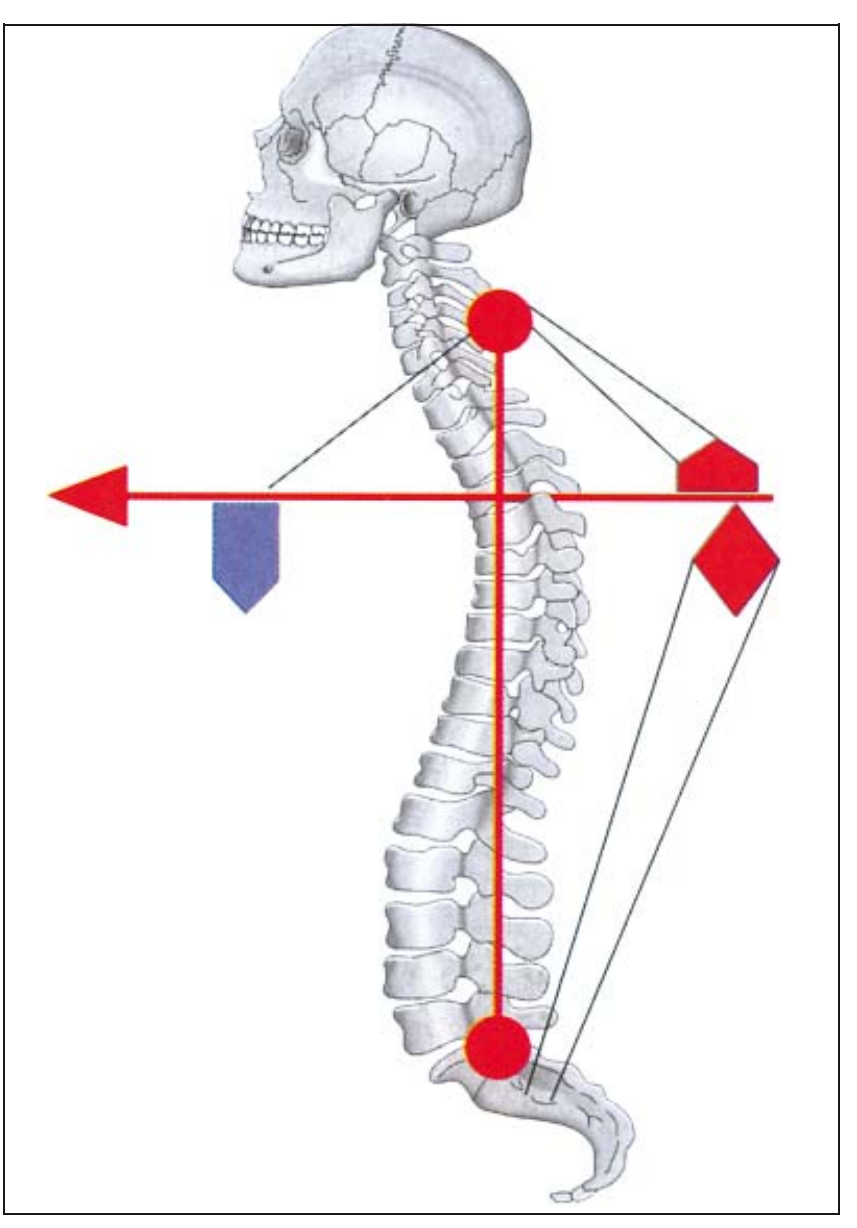

Abb. 1 Kranmodell der Wirbelsäule.

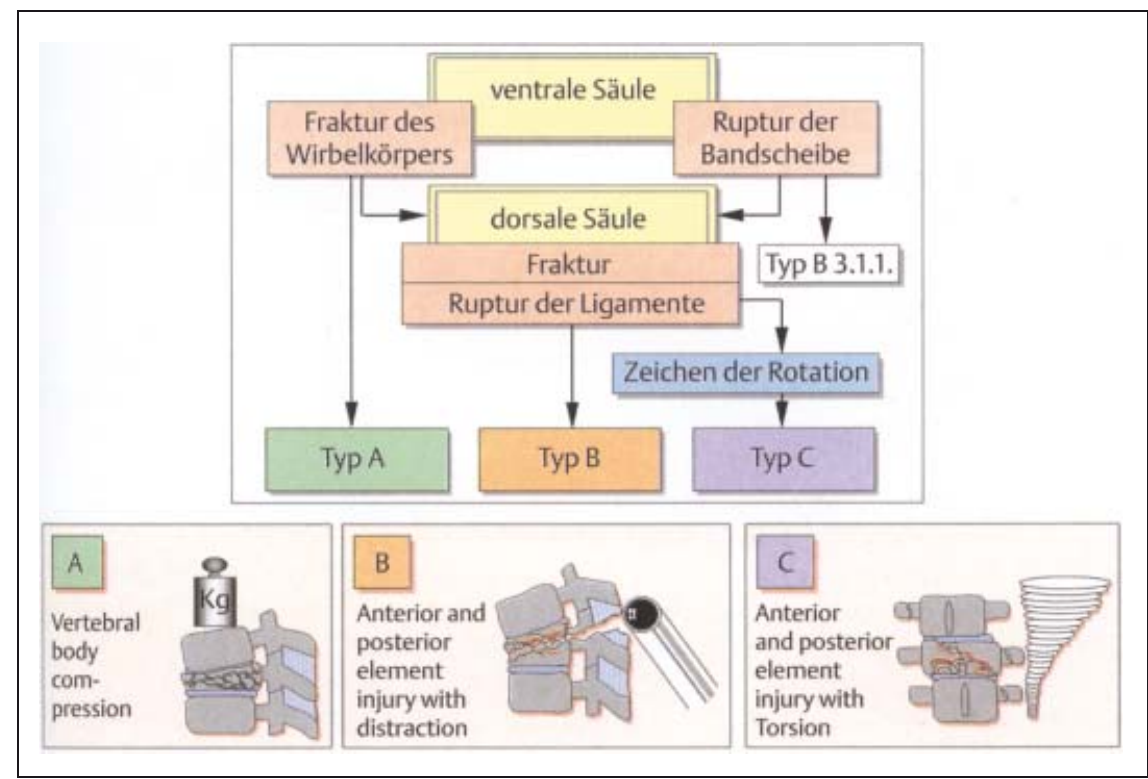

Abb.2 Klassifikation der Wirbelsäulenverletzung.

in der Wirbelsäule eingeleiteten Kraft dorsal innerhalb der Instrumentation erscheinen. Bei weiterhin bestehender ventraler Defektbildung kommt es zu einer
Wechselbiegebelastung der Instrumentation, die dann als Ursache für die Fehlergebnisse mit Lockerung der Verankerung in den Wirbeln, Korrekturverlusten und letztendlich Bruch des Instrumentationssystemes (z. B. der Pedikelschrauben) anzusehen ist.

Während für alleinige dorsale Spondylodesen im thorakolumbalen Bereich bei unterschiedlichen Indikationen Pseudarthroseraten von $13-82 \%$ beschrieben wurden [5,9] führen kombinierte dorsoventrale Spondylodesen zu signifikant besseren Endergebnissen. Die Pseudarthroserate liegt hier zwischen 4 und $8 \%$ $[9,10]$.

\section{Klassifikation - Indikation}

Auch in der Wirbelsäulentraumatologie gilt der Grundsatz, dass für eine erfolgreiche Behandlung zunächst eine exakte Diagnosestellung erfolgen muss, bevor eine erfolgversprechende Therapie eingeleitet werden kann. Der Heilverlauf einer Wirbelsäulenverletzung ist abhängig von Art und Ausmaß der Destruktion.

Erfahrungsgemäß zeigen knöcherne Verletzungen prinzipiell eine gute Heilungstendenz („temporäre Instabilität“), Bandscheiben und Bandverletzungen tendieren zu narbiger Defektheilung mit persistierender Instabilität $[7,1]$.

Wir kennen aber auch knöcherne und/ oder diskoligamentäre Verletzungsmuster die im späteren Verlauf zu progredienten kyphotischen, skoliotischen oder Rotationsfehlstellungen mit schmerzhaften Störungen der Wirbelsäulenstatik und sekundären neurologischen Spätschäden führen können $[2,3,4,12]$.

Die Differenzierung in stabile und instabile Verletzungen ist von entscheidender Bedeutung, sowohl für die konservative Therapie (frühfunktionell?, Immobilisationsdauer?, Repositionsverfahren?), als auch für die Indikation und Verfahrensweise operativer Behandlung. Nach zahlreichen Einteilungsvorschlägen hat sich heute zunehmend die 1994 publizierte Klassifikation von Magerl, Harms und Gertzbein durchgesetzt, die nach pathomechanischen Gesichtspunkten drei Hauptgruppen von Verletzungen definieren, die typischer Weise entstehen bei Dominanz von auf die Wirbelsäule einwirkenden Kompressions-, Distraktionsund Torsionskräften. Somit besteht die Klassifikation aus drei Hauptgruppen (Abb. 2).
A. Kompressionsverletzung
B. Distraktionsverletzung
C. Rotationsverletzung 


\section{Typ A - Kompressionsverletzung}

Ca. 65\% aller thorakolumbalen Wirbelsäulenverletzungen sind diesem Verletzungstyp zuzuordnen. Es handelt sich hierbei immer um eine Verletzung der vorderen Säule, dorsal kann eine Längsspaltung der Lamina entstehen, der dorsale Ligamentkomplex ist immer intakt.

\section{Typ B - Distraktionsverletzung}

Diese Verletzung ist gekennzeichnet durch eine Destruktion der hinteren Gliederkette, die in Kombination mit einer Verletzung der vorderen Säule auftreten kann. Die dorsale distraktionsbedingte Zerreißung kann transartikulär und transligamentär verlaufen oder knöchern durch die Gelenkfortsätze, den Bogen oder die Pedikel. Es ist somit nachvollziehbar, dass Typ-B-Verletzungen, entsprechend der Schädigung von vorderen und hinteren Strukturen der Wirbelsäule, wesentlich instabiler sind als TypA-Verletzungen. Seltener sind Distraktionsverletzungen der vorderen Säule.

\section{Typ C - Torsionsverletzungen}

Die Torsionsverletzungen weisen die Merkmale der Typ-A-Verletzung (Kompression) und der Typ-B-Verletzung (Distraktionsverletzung) in Kombination mit einer mehr oder weniger stark ausgeprägten Rotation auf. Häufig liegt hier eine mehrsegmentale Verletzung mit Frakturen von Querfortsätzen, Rippen oder Rippenluxationen, benachbart zum hauptverletzten Segment vor. Röntgenologisch ist besonders in der Frontalebene eine translatorische und rotatorische Versetzung der Wirbelsäule mit Verletzungen der Achse des Spinalkanales nachweisbar.

Die Typ-C-Verletzungen ist die instabilste Form aller drei Gruppen.

Noch vor wenigen Jahren wurde der überwiegende Anteil der Wirbelsäulenverletzungen, unabhängig von der Frakturklassifikation durch alleinige dorsale Verfahren behandelt. Der Rekonstruktion der hauptbelasteten vorderen Säule wurde somit nicht ausreichend Rechnung getragen, was sich in den Ergebnissen niederschlug. Die Indikation zur ventralen Stabilisierung instabiler Frakturen, insbesondere auch des thorakolumbalen Überganges stellt sich immer dann, wenn neben Verletzungen der dorsalen Elemente in der vorderen Säule ein erheblicher knöcherner Schaden einge- treten ist und wenn aufgrund des Ausmaßes der knöchernen und diskoligamentären Schäden ein nicht vertretbares Maß an Instabilität bei ausschließlich dorsaler Instrumentation verbleibt. Die ventrale Stabilisierung wird durch kortikospongiöse Späne oder tricortikale Beckenkammblöcke erreicht, hier wird zur Erhöhung der Stabilität ein antero-lateral angelegtes Implant empfohlen. Bei hochgradiger Zerstörung des Wirbelkörpers und erforderlicher partieller oder totaler Korporektomie kann die Kontinuität mittels Titan-Korb als Wirbelkörperersatz mit zusätzlicher Spongiosaanlagerung durchgeführt werden.

Zur Stabilisierung instabiler thorakolumbaler Frakturen hat sich, wenn ein ventrales Vorgehen als notwendig erachtet wird, eine Kombination aus dorsaler Instrumentierung und ventraler $\mathrm{Fu}-$ sion durchgesetzt, wobei überwiegend ein zweizeitiges Vorgehen empfohlen wird. Seltener wird ein ventro-dorsales Verfahren empfohlen. Aus Gründen der höheren Stabilität wird neben der Transplantation eines druckstabilen kortikospongiösen Spans eine zusätzliche ventrale Instrumentation empfohlen [11]. Bei Nichtbeachtung der biomechanischen Situation kann es auch bei der ventralen Instrumentation zu Schraubenbrüchen und Dislokationen kommen. Die Gesamtkomplikationsrate bei ausschließlich ventraler Instrumentation wird mit bis zu 20\% angegeben. Diese Rate sinkt deutlich bei kombinierter dorsaler und ventraler Stabilisierung.

Lediglich bei kombinierten dorsalen und ventralen Operationen lässt sich die primär erreichte Korrektur weitgehend halten.

Abhängig von der Frakturform wird auch bei ventralem Vorgehen die kürzest mögliche definitive Fusion gewählt. Frakturausläufer, die bis in den Grundplattenbereich laufen und hier keine wesentliche Frakturdistanz oder Dislokation zeigen, bedeuten nicht automatisch eine bisegmentale Fusion. Häufig ist auch hier noch eine monosegmentale Fusionierung zu vertreten, da nicht zwangsweise eine Zerstörung der darunter liegenden Bandscheibe vorliegt und somit eine Instabilität in diesem Segment nicht besteht.

Bei Mehretagenverletzungen der Brustund Lendenwirbelsäule sind gelegentlich auch längerstreckige dorsale Instrumentierungen in Kombination mit mono- oder bisegmentalen ventralen Fusionen erforderlich.

\section{Ventrale Zugänge}

Im Vergleich zur dorsalen Instrumentierung ergeben sich für alle ventralen Verfahren höhere operationstechnische Anforderungen, Risiken, höhere Patientenbelastung und letztendlich - zumindestens bis zur Einführung minimal invasiver Verfahren - eine deutlich erhöhte Zugangsmorbidität.

So ist bei polytraumatisierten Patienten oder Patienten in reduziertem Allgemeinzustand das unvermeidbare Operationstrauma eines ventralen Eingriffes erst nach Besserung des Allgemeinzustandes - wenn überhaupt - möglich.

Bei Verlegung des Spinalkanales kann von einem vorderen Zugang eine Enttrümmerung des Spinalkanales stattfinden, da man sich hier direkt am Hauptort der Verletzung befindet.

Entsprechend der erforderlichen operativen Darstellung der zu instrumentierenden Wirbelsäulenregion sowie der Höhe der Verletzung erfolgt der Zugang in Seitenlage, entweder transthorakal, retroperitoneal oder kombiniert im Sinne einer Lumbophrenotomie (Abb. 3).

Prinzipiell ist der Zugang zur Wirbelsäule bds. möglich, wobei wir oberhalb Th 8 einen rechtsseitigen Zugang, tiefer Th 8 einen linksseitigen Zugangsweg bevorzugen.

Über die Hälfte der Wirbelkörperverletzungen im Brust- und Lendenwirbelsäulenbereich befinden sich im thorakolumbalen Übergang, betroffen sind vorwiegend BWK 12 und LWK 1.

\section{Thorakotomie}

Eine Thorakotomie zur Darstellung der BWS kann sowohl von re. als auch von li. durchgeführt werden. Eine Intubation mit Doppellumentubus ist hilfreich. Die obere BWS bis in Höhe des 4.Brustwirbelkörpers ist operationstechnisch schwierig zu erreichen. Eine exakte Operationsplanung ist erforderlich.

Entweder kommt hier ein Zugang mit Sternotomie infrage oder der Zugang wird erreicht über einen postero-lateralen Zugang mit Resektion der Rippe (Kostotransversektomie). 


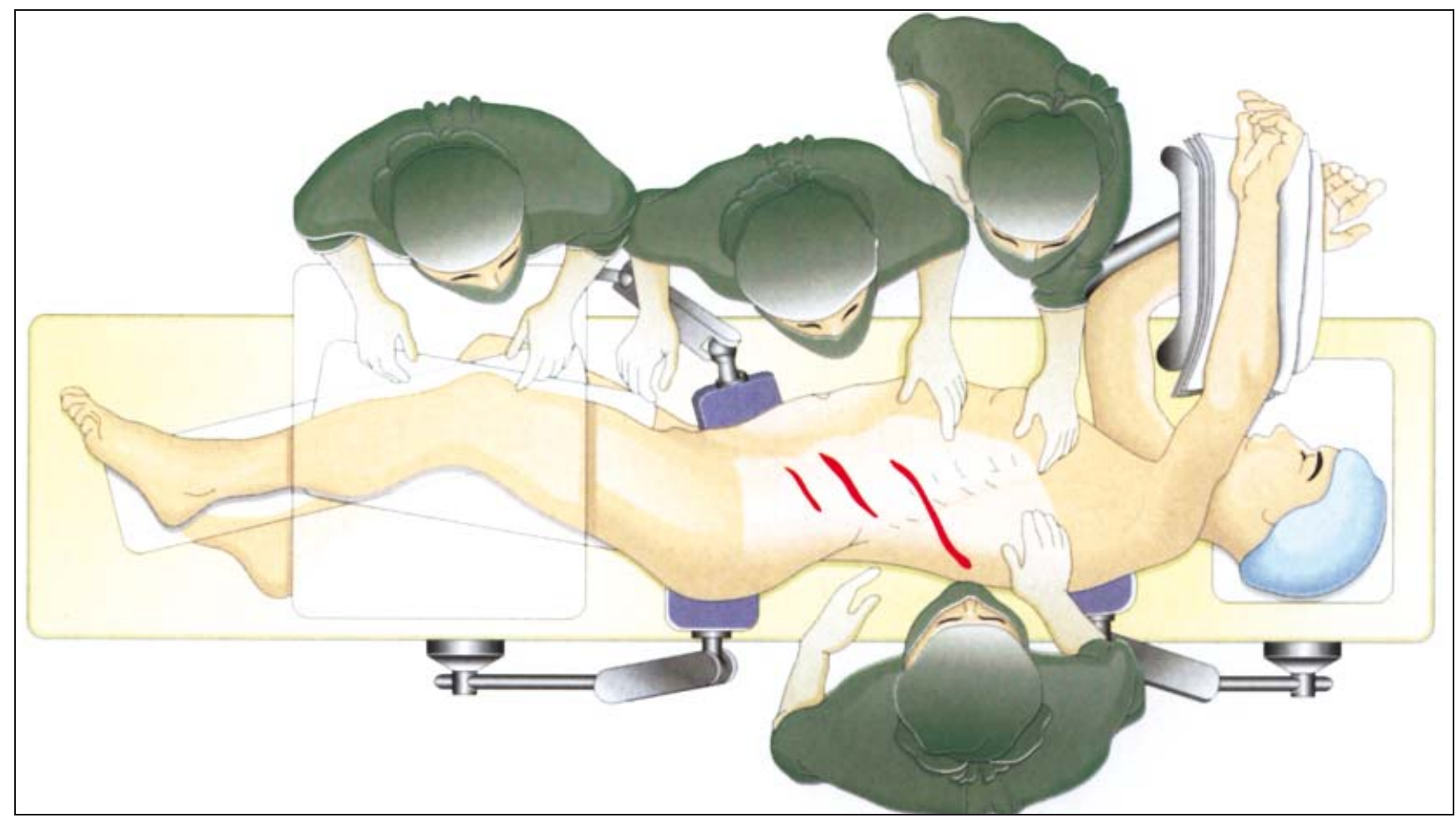

Abb.3 Seitenlage zur Versorgung der ventralen Säule. 1) thorakal 2) lumbal 3) Beckenkamm.

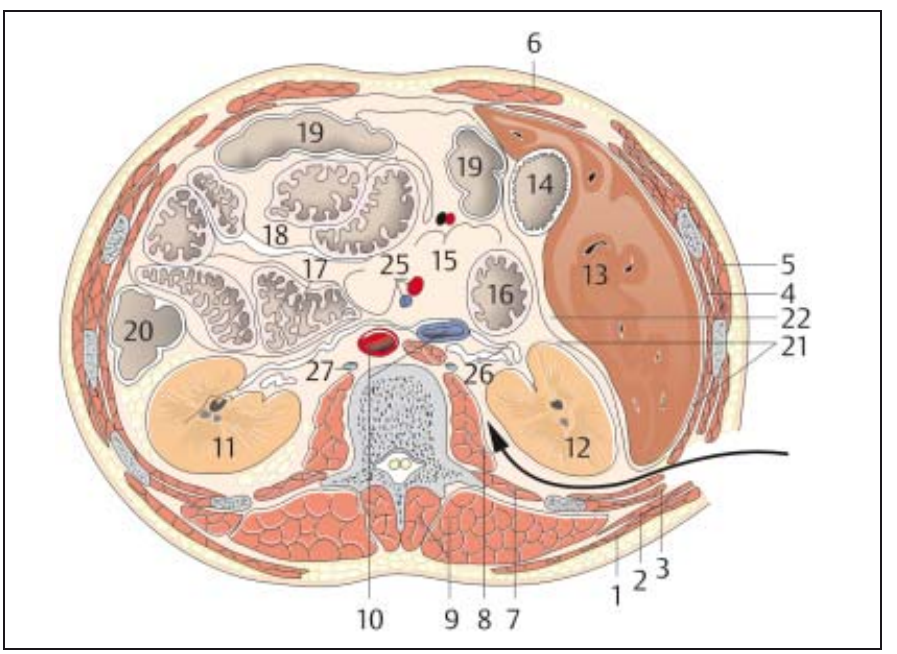

Abb.4 Retroperitonealer Zugang zur Lendenwirbelsäule von rechts. $1 \mathrm{M}$. latissimus dorsi $2 \mathrm{M}$. serratus posterior inferior $3 \mathrm{Mm}$. intercostales 4 Diaphragma thoracis 5 laterale Bauchmuskulatur $6 \mathrm{M}$. rectus abdominis 7 M. quadratus lumborum $8 \mathrm{M}$. psoas major 9 autochthone Rückenmuskulatur 10 Diaphragma thoracis, Crura medialia 11 Ren sinister 12 Ren dexter 13 Lobus dexter hepatis 14 Vesica fellea 15 Pancreas 16 Pars descendens duodeni 17 Flexura duodenojejunalis 18 Jejunum 19 Colon transversum 20 Colon descendens 21 Peritoneum parietale 22 Cavitas peritonealis 23 Pars abdominalis aortae $24 \mathrm{~V}$. cava interior 25 Vasa mesenterica superiora 26 Vasa renalia dextra 27 Truncus sympathicus II Vertebra lumbalis.

\section{Thorakolumbophrenotomie}

Für Verletzungen des thorakolumbalen Überganges wurde traditionell der transpleural retroperitoneale Zugang nach Hodgson benutzt (Abb.4). Dieser Zugang lässt eine langstreckige Freilegung der Wirbelsäule zu. Es handelt sich hier um einen ausgedehnten Zugang mit Durchtrennung der Bauchdeckenmuskulatur in Faserrichtung. Eröffnung des Brustkorbes oberhalb der 10. Rippe (evtl. Rippenresektion). Nach Abschieben des Peritoneums und der retroperitonealen Organe vom Zwerchfell wird dies bogenförmig, etwa $1,5 \mathrm{~cm}$ vom Rippenansatz entfernt, durchtrennt und abgelöst. Vorgelegte Haltefäden dienen als Orientierung für die spätere Refixation. Über diesen $\mathrm{Zu}$ gang kann die Wirbelsäule von Th 9-L 5 freigelegt werden.

Dieser ausgedehnte Zugang wird zunehmend durch endoskopische Verfahren hauptsächlich bei Verletzungen ab Th 8-L 1/2 abgelöst.

\section{Lumbotomie}

Ab LWK 2 kann ein alleiniger retroperitonealer Zugang, der ebenfalls eine Durchtrennung der Bauchmuskulatur erfordert zum Einsatz kommen. Der Zugang verläuft schräg in der Mitte zwischen Rippenbogen und Beckenkamm, um die Bauchwandinnervation so wenig wie möglich zu schädigen. Der Peritonealsack wird vom Muskulus transversus abdominis und quadratus lumborum nach medial abpräpariert.

Bei kräftigem Muskulus psoas kann sich die Abpräparation dieses Muskels von der seitlichen Wirbelsäule als aufwändig erweisen. $\mathrm{Zu}$ schonen sind die im $\mathrm{Zu}$ gangweg verlaufenden Nerven, wie der Nervus ilio hypogastrikus, Nervus ilio 

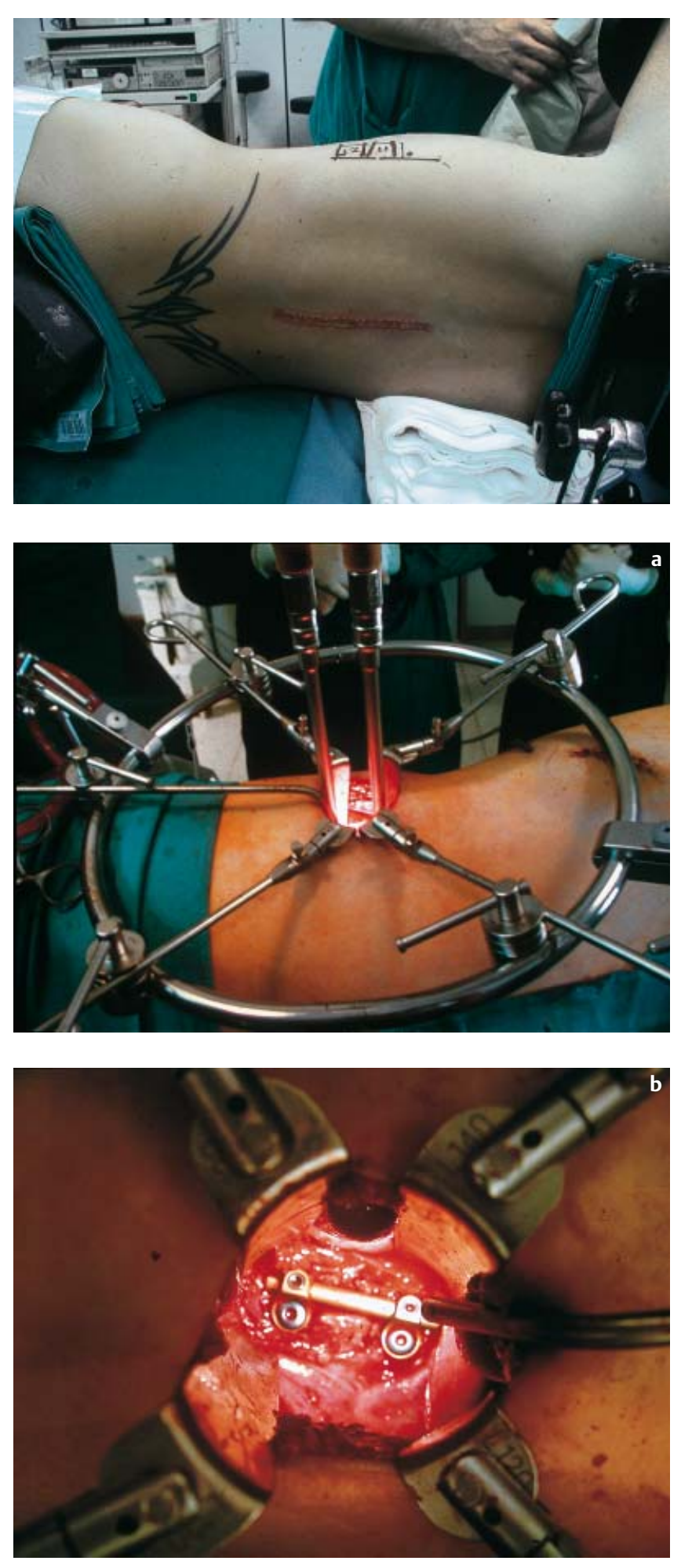

Abb. 5 Seitenlage, unter Bildwandlerkontrolle angezeichnete Frakturhöhe.

Abb. 6 (a) Eingesetztes Retraktorsystem (Syntrame) (b) OP-Feld, eingebrachter Beckenkammblock und VentrofixEinstabmontage. inguinalis, Nervus cutanaeus femoris lateralis und der 12. Interkostalnerv.

\section{Pararektaler retroperitonealer Zugang zu den Segmenten L 4/5 und L 5/S 1}

Der Patient liegt in Rückenlage mit leicht gebeugtem Hüft- und Kniegelenk zur Entspannung der Bauchmuskulatur. Der Zugang kann von re. und li. erfolgen. Bei diesem Zugang muss keine Bauchmuskulatur durchtrennt werden. Nach Durchtrennung der Rektusscheide erfolgt stumpfes Abschieben des Peritoneums von der seitlichen Bauchwand und vom Muskulus quadratus lumborum und Psoas bis zur Gefäßbifurkation. Im Bereich des lumbosakralen Überganges ist auch ein transperitonealer Zugang möglich.

\section{Minimal invasive Zugänge}

Wegen der Zugangsmorbidität - zum Beispiel verbleibende Bauchwandschwäche durch Schädigung der hier segmental einstrahlenden Nerven - vorwiegend im thorakolumbalen Übergangsbereich wurden in den letzten Jahren zunehmend minimal-invasive Zugänge entwickelt ob offen oder endoskopisch - mit erheblicher Reduktion des Weichteiltraumas. Parallel hierzu erfolgte auch die Entwicklung neuerer Implantate. Diese Verfahren setzen eine exakte präoperative und intraoperative Planung voraus, da sowohl bei endoskopischen Verfahren, als auch bei minimal invasiven Verfahren senkrecht zum Wirbelkörper instrumentell gearbeitet werden muss. Dies bedeutet, dass vor Beginn der Operation eine genaue Höhenlokalisation mit Aufzeichnung der entsprechenden Wirbelkörper unter Bildwandlerkontrolle erfolgt (Abb.5).

Nach entsprechender Anzeichnung der Höhe erfolgt die Durchtrennung der Haut und Muskulatur direkt über dem frakturierten Wirbelkörper. Es sind hier nur kleine Zugänge von 6-8 cm erforderlich.

Zum Offenhalten des Zugangsweges werden spezielle Retraktoren (z.B. Synframe) eingesetzt (Abb.6).

Zunehmend gewinnen endoskopische (thorakoskopische) Verfahren an Verbreitung. Hierzu sei auf den Beitrag „Minimal-invasive und endoskopische Technik an Brust- und Lendenwirbelsäule" verwiesen. 

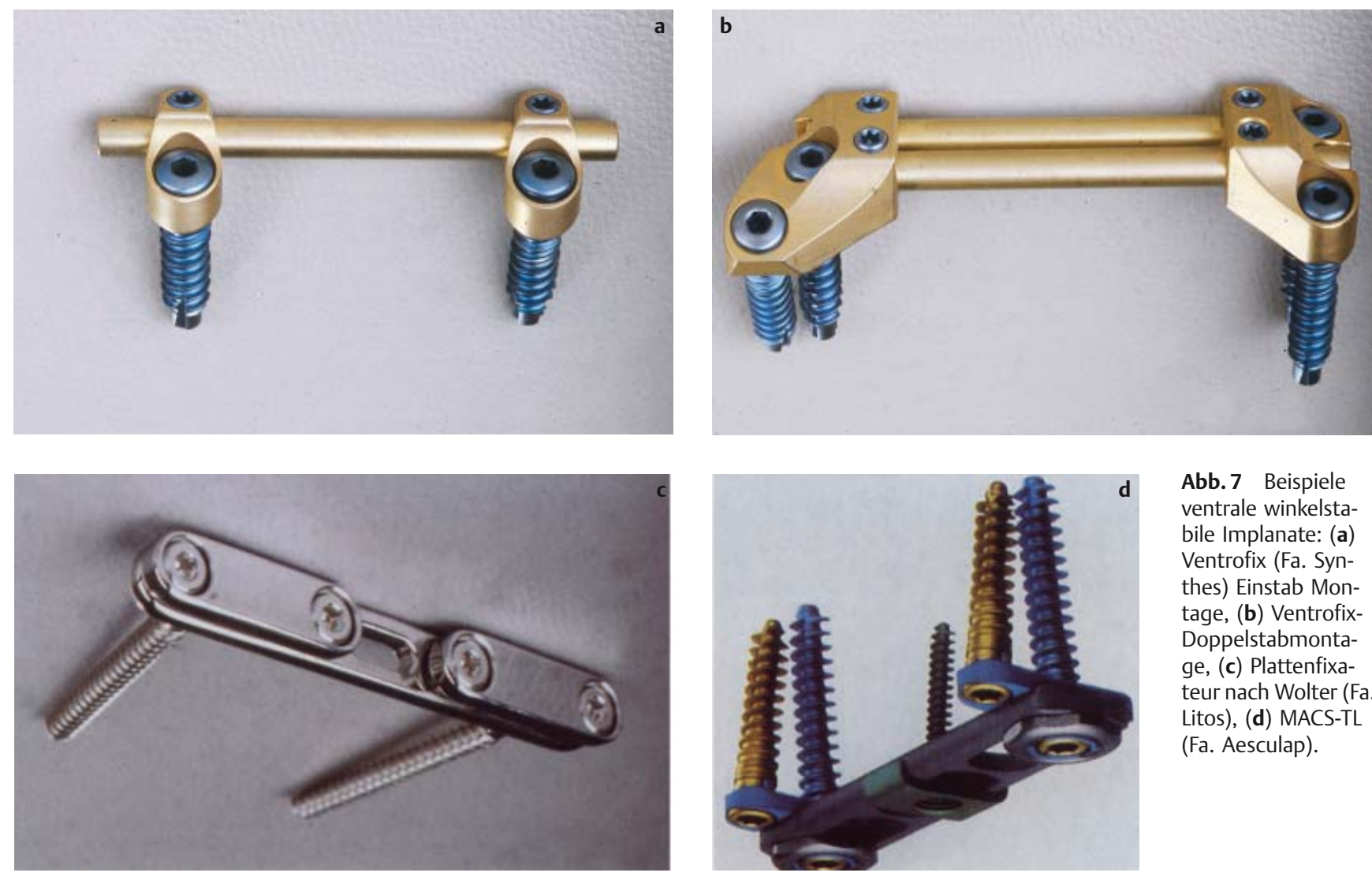

Abb. 7 Beispiele ventrale winkelstabile Implanate: (a)

Ventrofix (Fa. Synthes) Einstab Montage, (b) VentrofixDoppelstabmontage, (c) Plattenfixateur nach Wolter (Fa. Litos), (d) MACS-TL

(Fa. Aesculap).

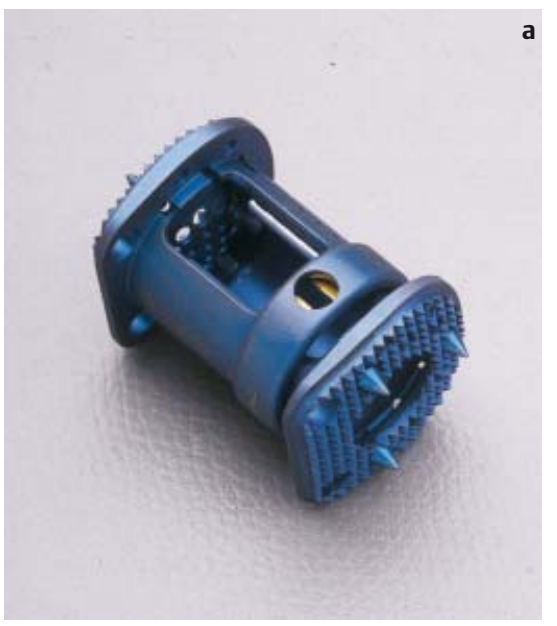

\section{Operatives Vorgehen}

Implantate

Bei der rasanten Entwicklung der Wirbelsäulenchirurgie sind Anzahl und technische Weiterentwicklung der zur Verfügung stehenden Implantate auch für den erfahrenen Operateur kaum noch zu überblicken.
Nach anfänglichem Einsatz normaler Osteosyntheseplatten kommen heute vorwiegend winkelstabile Ein- oder Zweistabsysteme sowie spezielle winkelstabile Plattenkonfigurationen zum Einsatz (Abb. 7).

Bei höhergradiger Zerstörung des Wirbelkörpers und erforderlichem Spinal-
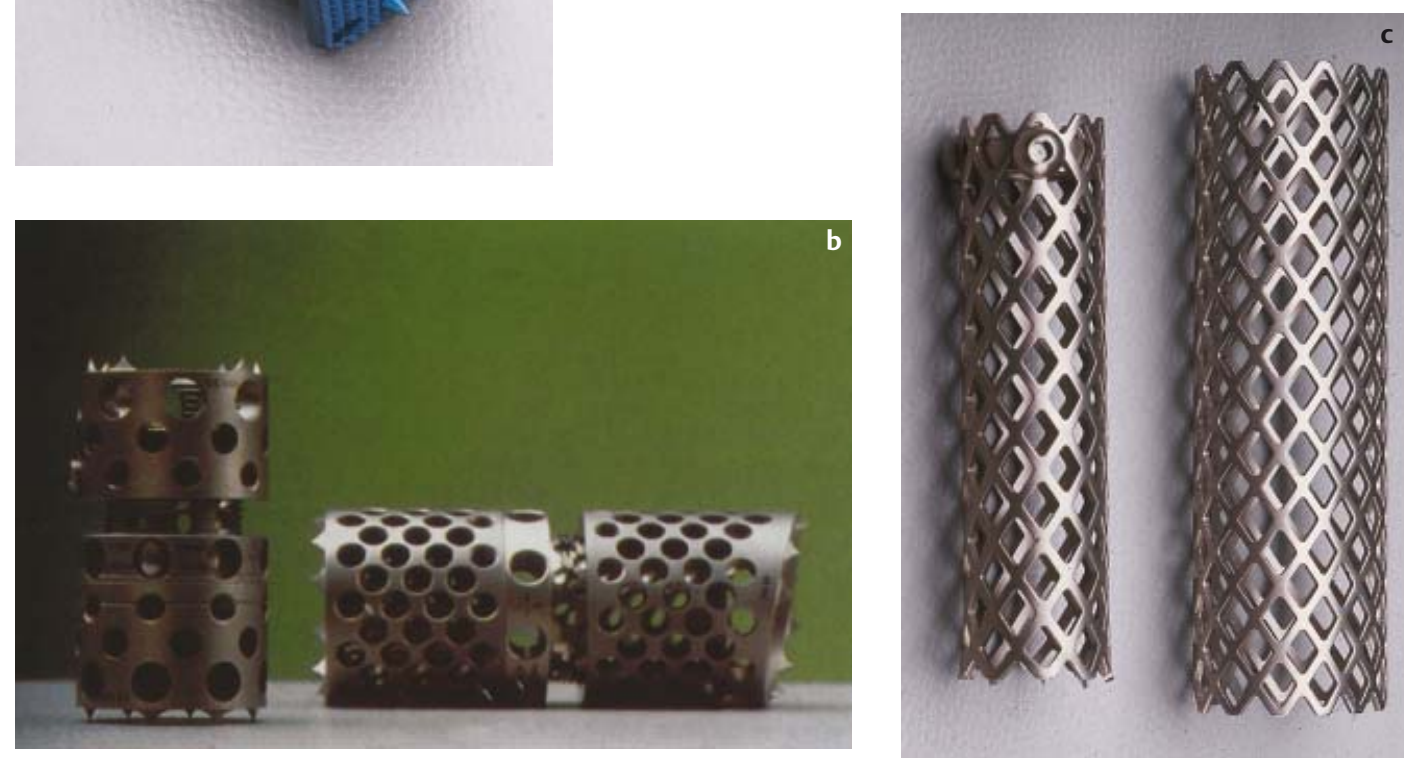

Abb. 8 Wirbelkörperersatz (a) Synex

(Fa. Aesculap), (b)

VBR (Fa. Ulrich), (c)

MOSS-Titankorb zuschneidbar. 
kanalclearing mit Teilkorporektomie und Ausräumung der Wirbelkörperhinterkanten kommen zunehmend, vorwiegend aus Titan bestehende - Wirbelkörperersatzimplantate in Kombination mit einer Spongiosaauffüllung oder Spongiosaplastik zum Einsatz (Abb.8).

Die Möglichkeiten einer intraoperativen Expandierbarkeit über ein Gewinde oder sonstige Aufspreizmechanismen sind häufig vorhanden.

Somit kann über die eingebrachten winkelstabilen Implantate oder expandierbare Cages eine Rekonturierung des Wirbelsäulenprofiles erreicht werden.

\section{Reposition}

Wir bevorzugen ein zweizeitiges Vorgehen bei der Versorgung instabiler Wirbelsäulenverletzungen. In der Regel kann durch eine dorsale Instrumentierung notfallmäßig die Reposition vorgenommen werden, eine kyphotische Fehlstellung kann in Bauchlage am effektivsten aufgerichtet werden. Bei relevanter Einengung des Spinalkanales kann eine ausreichende Wiederherstellung der Spinalkanalweite durch Einsatz der Ligamentotaxis gelingen, gegebenenfalls kann über eine dorsale Entlastung ein ausreichendes Spinalkanalclearing durchgeführt werden.

Bei kompletter Verlegung des Spinalkanales kann bei ausreichendem Allgemeinzustand des Unfallverletzten ein einzeitiges dorso-ventrales Vorgehen notwendig sein. In der Regel kann jedoch die ventrale Operation elektiv durchgeführt werden.

Die Aufrichtung einer kyphotischen Fehlstellung in Seitenlage ist schwierig. Hilfreich sind hier entweder entsprechend lange oder großübersetzende Spondylodesespreizer. Hilfsweise kann auch die in der Beckenchirurgie bewährte Jungbluth-Zange über eingebrachte Schrauben im Nachbarwirbel eingesetzt werden (Abb. 9).

Expandierbare Cages oder winkelstabile Implantate lassen eine Distraktion über das Implantat zu.

\section{Spinale Dekompression}

Brust- und Lendenwirbelsäule verhalten sich bezüglich des Reserveraums für das Rückenmark unterschiedlich.

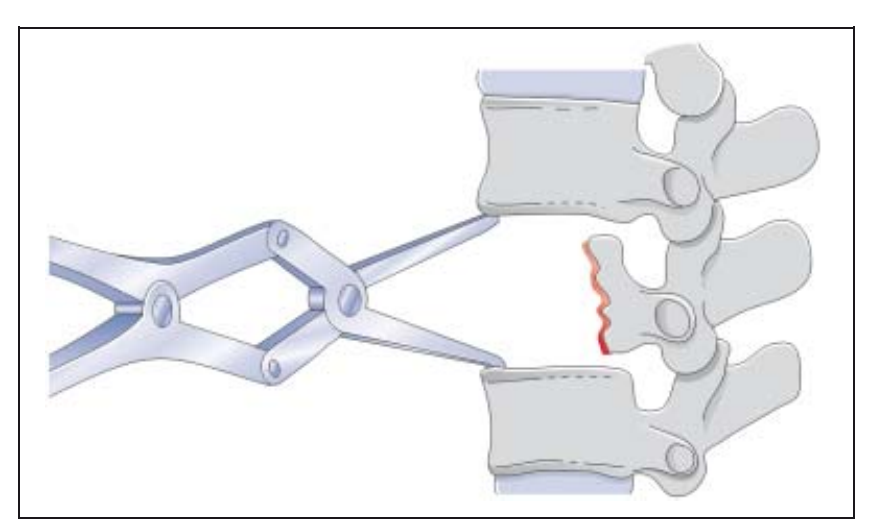

Abb. 9 Aufrichtung Wirbelkörper mit Spondylodesespreizer.
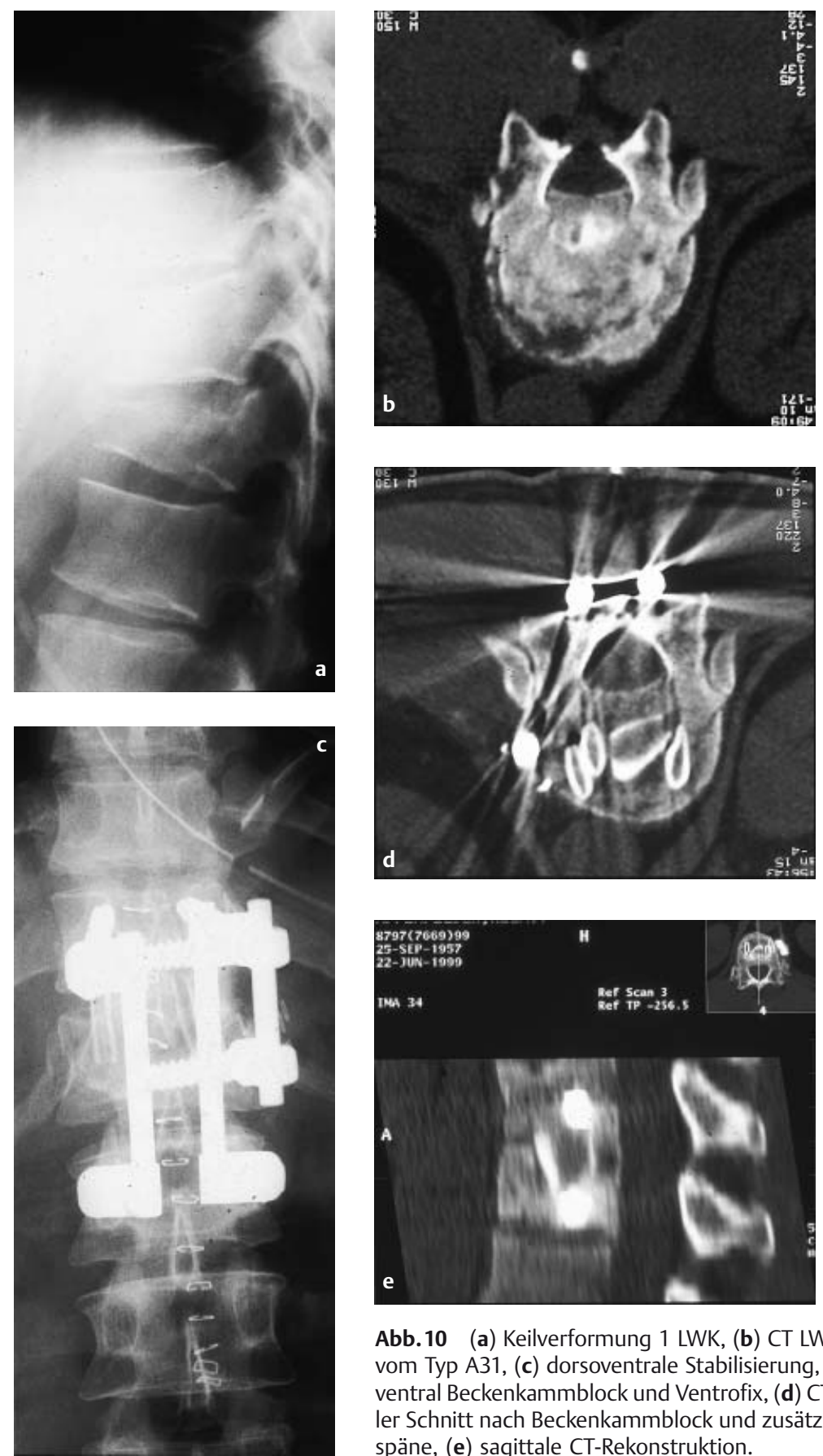

Abb.10 (a) Keilverformung 1 LWK, (b) CT LWK 1 Fraktur vom Typ A31, (c) dorsoventrale Stabilisierung, dorsal USS, ventral Beckenkammblock und Ventrofix, (d) СT horizontaler Schnitt nach Beckenkammblock und zusätzlich Rippenspäne, (e) sagittale CT-Rekonstruktion. 

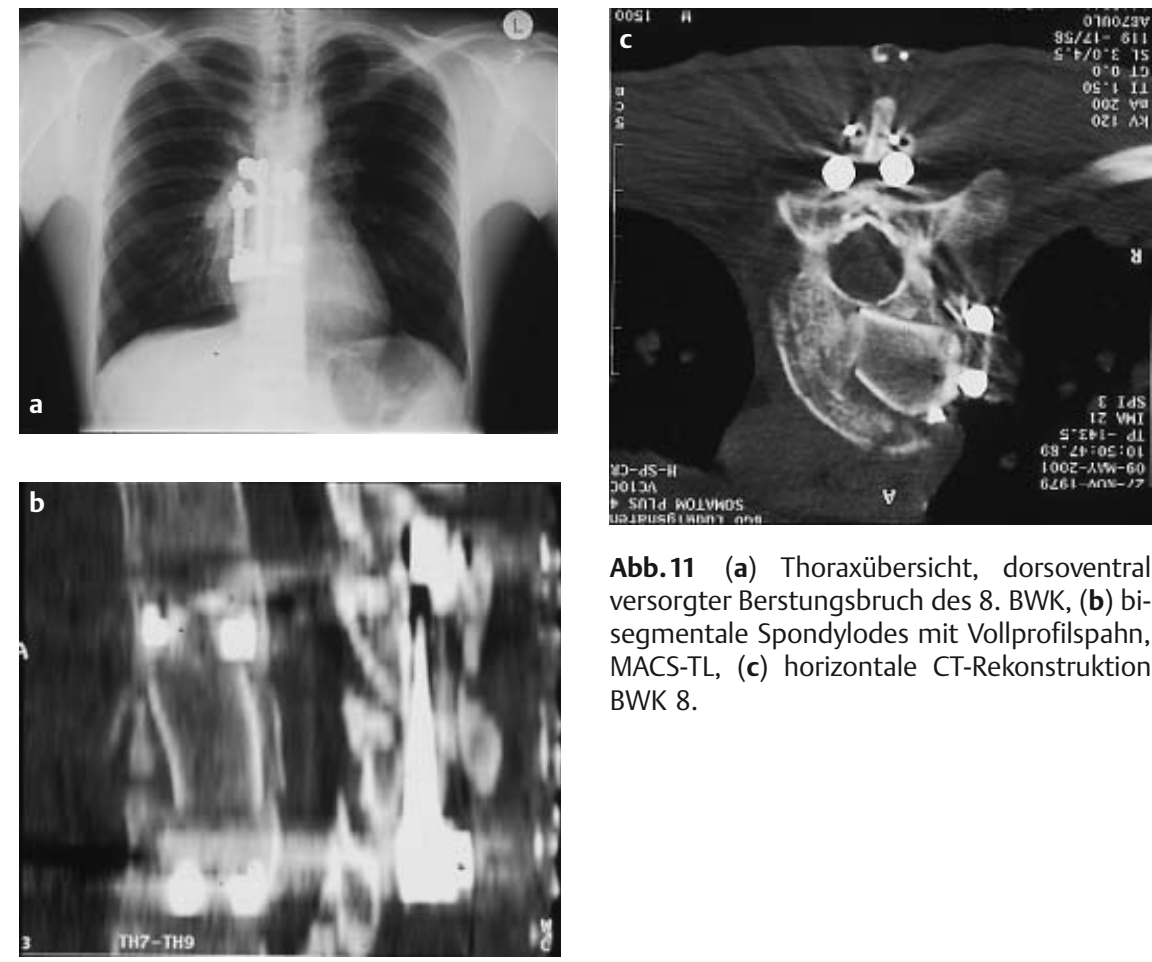

Abb.11 (a) Thoraxübersicht, dorsoventral versorgter Berstungsbruch des 8 . BWK, (b) bisegmentale Spondylodes mit Vollprofilspahn, MACS-TL, (c) horizontale CT-Rekonstruktion BWK 8.
Während im Bereich der Brustwirbelsäule bereits bei einer Einengung von $20 \%$ mit akuten oder sich chronisch entwickelnden neurologischen Ausfällen zu rechnen ist, können im Lendenwirbelsäulenbereich Verlegungen bis zu 50\% ohne neurologische Defizite einhergehen.

Methodisch bestehen mehrere Möglichkeiten der Dekompression zur Verfügung.

Neben der Dekompression durch indirekte Reposition eines dislozierten Wirbelkörperhinterkantenfragmentes durch die so genannte Ligamentotaxis bei dorsalem Vorgehen, besteht die Möglichkeit ebenfalls von dorsal eine direkte transspinale instrumentelle Reposition nach partieller Laminekomie durchzuführen.

Bei geplantem ventralen Vorgehen ist eine Dekompression des Rückenmarkes und der Cauda equina durch einen ventralen Zugang mit besserer Sicht und besserer Möglichkeit der instrumentellen
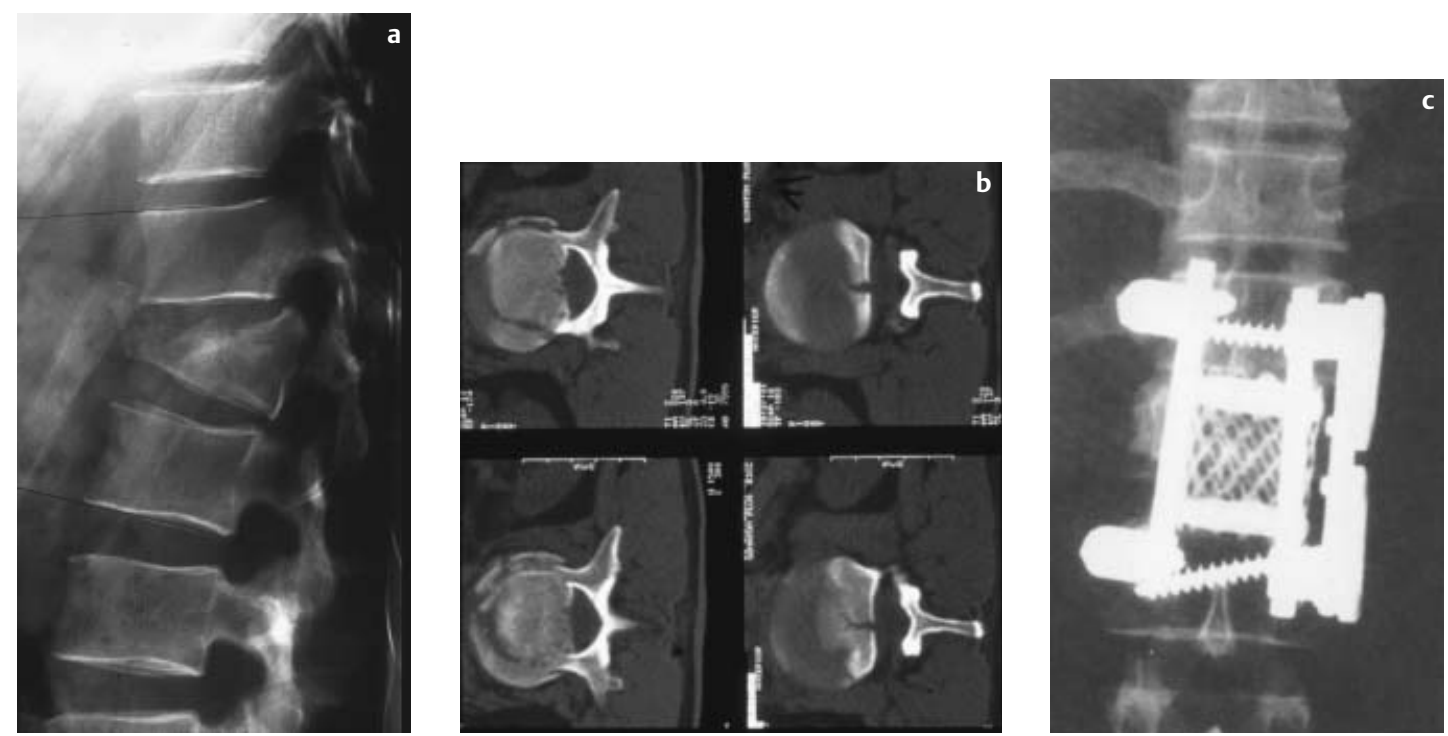

Abb.12 Kompressionsfraktur Typ A31 1 LWK (a) Nativröntgen (b) CT mit Hinterkanten Beteiligung (c/d) dorsoventrale Spondylodese mit Moss-Korb, zusätzlich ventral Wolter-Platte (e) horizontaler CT-Schnitt nach partieller Korporektomie und Implantation MOSSKorb (f) Entfernung dorsale Instrumentierung nach 6 Monaten.
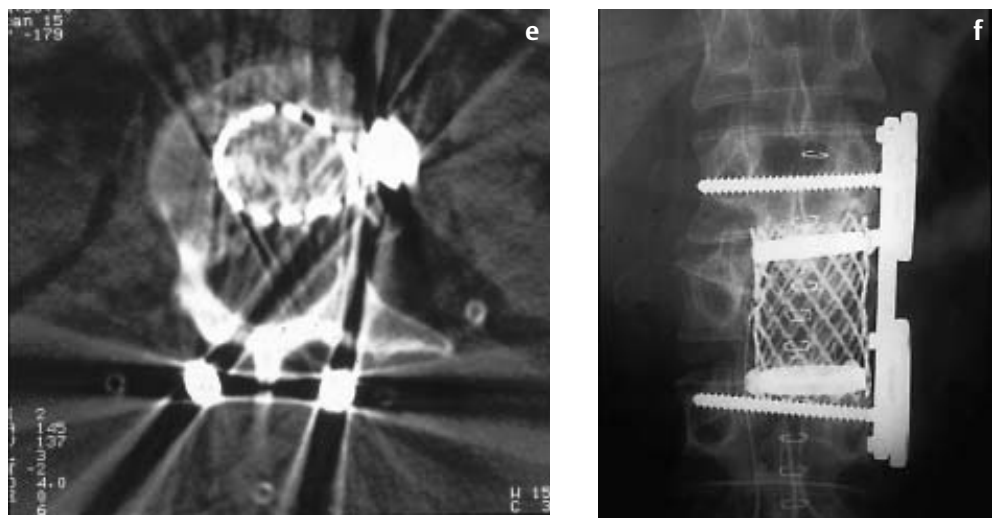

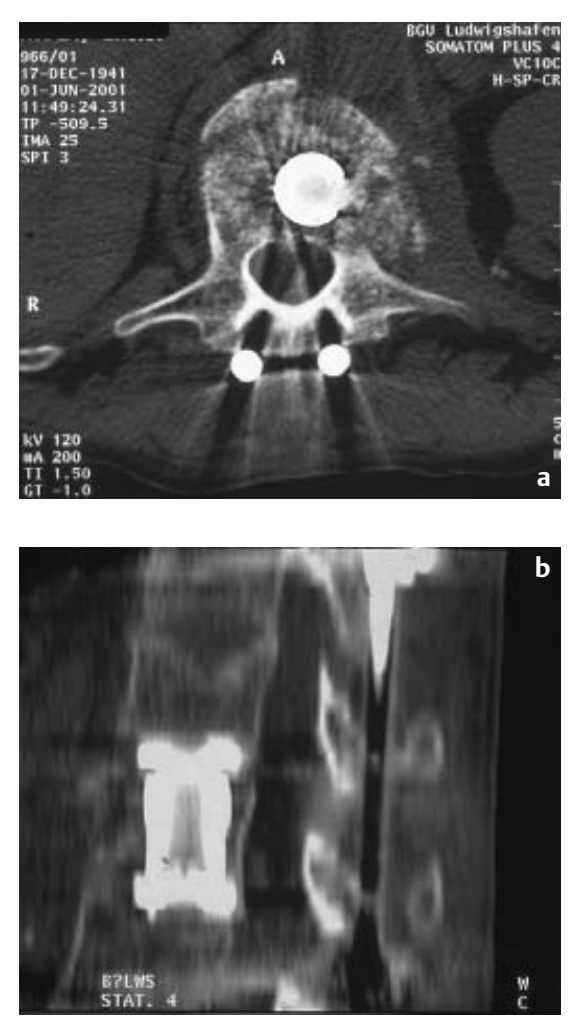

Abb.13a u. b horizontaler CT-Schnitt nach Implantation Synex mit seitlich angelagerter Spongiosa, saggitale CT-Rekonstruktion.
Manipulation gegenüber einer dorsalen Dekompression erheblich erleichtert.

Eine sorgfältige Präparation ist zur Vermeidung zusätzlicher Rückenmarkverletzungen oder auch zur Vermeidung schwer stillbarer Blutungen aus dem epiduralen Plexus erforderlich.

Vor allem bei der Extraktion von Fragmenten aus dem Spinalkanal können hier heftige Blutungen aus dem zerrissenen epiduralen Plexus entstehen, die gelegentlich nur mühsam durch Collagenflies und temporäre Tamponaden gestillt werden können.

\section{Rekonstruktion der ventralen Säule}

Ziel der ventralen Rekonstruktion ist eine völlige Wiederherstellung der Belastbarkeit der vorderen Säule, dies bedeutet, dass auf Dauer unabhängig von den eingebrachten Implantaten und Wirbelkörperersatzmaterialien eine knöcherne Überbrückung - je nach Indikations-
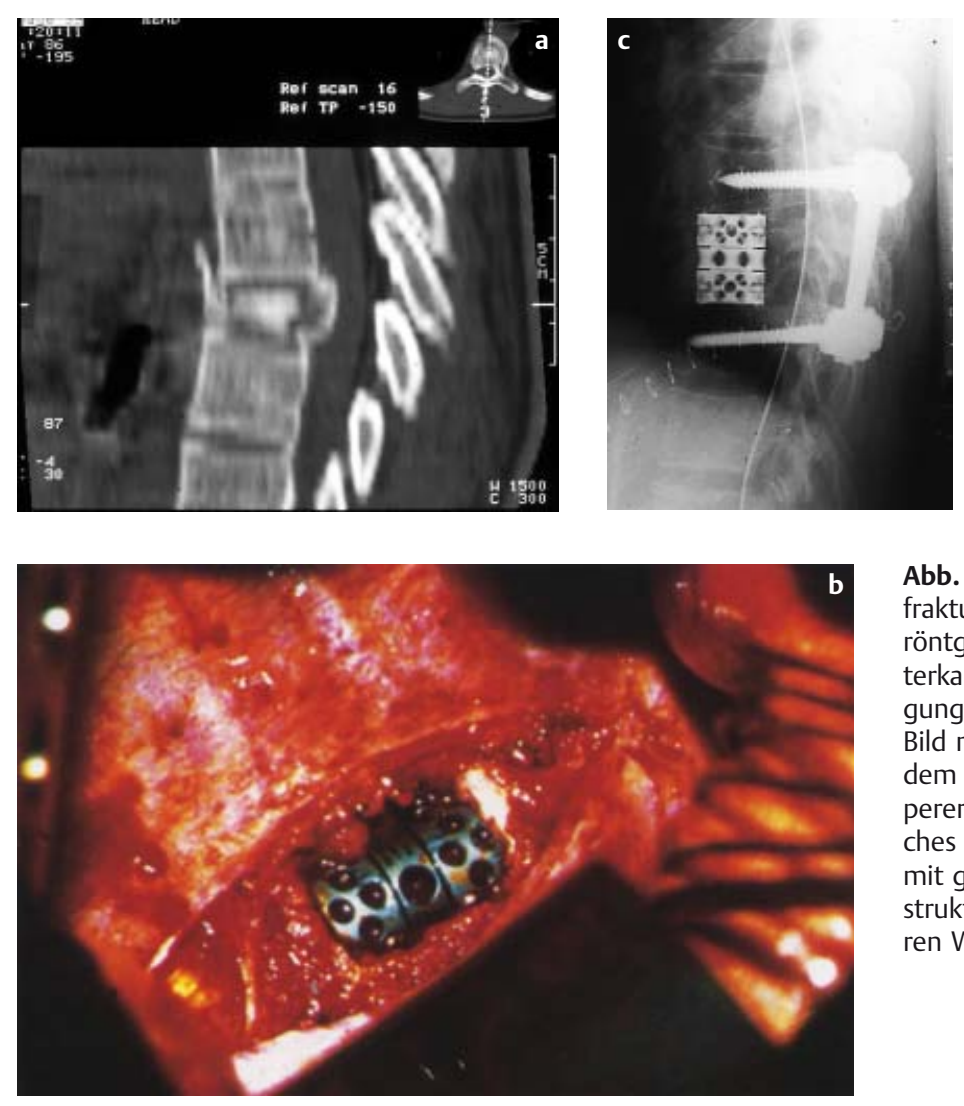

Abb.14 Berstungsfraktur (a) Nativröntgen zeigt Hinterkantenbeteiligung, (b) klinisches Bild mit Einliegendem VBR-Wirbelkörperersatz, (c) seitliches Röntgenbild mit guter Rekonstruktion der vorderen Wirbelsäule. stellung - von zwei oder mehreren benachbarten Wirbelkörpern erforderlich ist.

Vorzugsweise erfolgt deshalb der Aufbau der ventralen Säule durch Interposition eines biologisch hochwertigen trikortikalen Beckenkammblockes. Bei größeren Entnahmedefekten am Beckenkamm sollte zur Konturierung beispielsweise eine Titan-Drittelrohrplatte aufgebracht werden.

Ist die Frakturregion bereits durch eine winkelstabile Instrumentation dorsalseitig aufgerichtet und gesichert, kann die Fusion alleine durch Einbringen eines solchen Blockes herbeigeführt werden ohne zusätzliche ventrale Instrumentation.

Hier bietet sich eine spezielle Inlay-Technik an, bei der der trikortikale Vollprofilspan über eine entsprechend vorbereitete Nut in die Anschlusswirbelkörper eingebolzt wird (Abb.10). Wichtig ist, dass der zu interponierende Vollprofilspan bis über die Mittellinie hinaus eingebolzt wird, ähnlich der Nut- und Federtechnik des Zimmermannes.

Voraussetzung einer solchen Technik ist, dass die Fraktursituation keine spinale Dekompression erforderlich macht. Aus Stabilitätsgründen bevorzugen wir auch in diesen Fällen die Kombination mit einem winkelstabilen ventralen Implantat. Dieses Vorgehen eignet sich vorwiegend für monosegmentale Versorgungen im thorakolumbalen Übergangsbereich und Lendenwirbelsäulenbereich. Auch bisegmentale Stabilisierungen sind möglich (Abb.11).

Bei hochgradiger Zerstörung des Wirbelkörpers und der Notwendigkeit der Korporektomie kommen auch Wirbelkörperersatzimplantate, wie das z.B. mit Spongiosa gefüllte MOSS-Körbchen (Abb.12) zum Einsatz. Eine Kombination mit einem zusätzlichen Implantat ist hier nicht erforderlich, da bis zum Einwachsen der Spongiosa der Cage die Funktion einer Instrumentation übernimmt. Eine knöcherne Überbrückung ist auch hier für den Dauererfolg ausschlaggebend.

Auch bei den massiveren Implantaten (expandierbarer Wirbelkörperersatz Synex TM Fa. Synthes), aufdrehbarer Wirbelkörperersatz VBR (Fa. Ulrich) ist eine Spongiosaplastik erforderlich, um eine definitive knöcherne Fusion zu erreichen (Abb.13 u.14). 
Im eigenen Krankengut konnten wir regelhaft eine entsprechende knöcherne Überbrückung computertomographisch nachweisen.

Dessen ungeachtet sind auch hier gelegentlich leichtere Korrekturverluste zu verzeichnen.

Wesentlich ist beim Einsatz von Wirbelkörperersatzimplantaten an den benachbarten Wirbeln eine intakte Grundund Deckenplatte zu belassen, die lediglich angefrischt wird bis Blutpunkte zutage treten. Bei Verletzungen der Wirbelkörperabschlussplatten kommt es sonst zum Einsinken des Implantates in die Wirbelkörperspongiosa und damit zum Korrekturverlust. Von den meisten Implantaten fehlen allerdings bisher mittelund langfristige Ergebnisse.

\section{Literatur}

${ }^{1}$ Böhler J. Verletzung der Wirbelsäule - operative Behandlung, Indikation und Technik Z. Orthop 1974; 112: 894

${ }^{2}$ Böhm H, Harms J, Donk R, Zielke K. Correction and stabilization of angular kyphosis. Clin Orthop 1990; 259: 304-310

${ }^{3}$ Eysel P, Rompe JD, Hopf C, Meining G. Significance of the disk in failed reduction of surgically stabilized fractures of the truncal spine. Unfallchirurg 1994; 97: 451-457

${ }^{4}$ Harms J. Wann besteht die Indikation zur operativen Behandlung von Wirbelfrakturen? Langenbecks Arch Chir 1922; 377: $125-130$

${ }^{5}$ Hopf CA, Wacker L, Heine J. Analyse von Fehlschlägen bei der operativen Behandlung lumbosacraler Spondylodesen. Orthop Praxis 1993; 19: 149-153

${ }^{6}$ Knop C, Blauth M, Bastian L, Lange U, Kesting J, Tscherne H. Frakturen der thorakolumbalen Wirbelsäule - Spätergebnisse nach dorsaler Instrumentierung und ihre Konsequenzen. Unfallchirurg 1997; 100: 630-639

${ }^{7}$ Louis R. Les theories de l'instabilite. Rev Chir Orthop 1977; 63: 423-425

${ }^{8}$ Matthis, Biedermann L. Biomechanical analysis of the load sharing principle using TSRH and the MOSS-device. Posterexhibition Int. Meeting on advanced Spine Techniques. Munich 1996

${ }_{9}^{9}$ Matschke S. Behandlungsergebnisse operativ versorgter traumatischer Wirbelsäulenverletzungen im Zeitraum 1986-1990 unter
Anwendung der Frakturklassifikation nach Magerl, Harms, Gertzbein, Aebi und Nazarian und der daraus abgeleiteten operativen Technik. Inauguraldissertation der medizinischen Fakultät des Saarlandes. 1999

${ }^{10}$ Strempel AV. Dorsoventrale Fusion im Bereich der unteren Stammwirbelsäule. In: Die Instabilität des lumbosakralen Scharnieres. Georg Thieme Verlag Stuttgart-New York 1992; 245-250

${ }^{11}$ Whitesides TE. Traumatic kyphosis of the thoracolumbar spine. Clin Orthop 1977; 128: $78-92$

${ }^{12}$ Winkler H. Der Korrekturverlust nach operativer Stabilisierung thorakolumbaler Wirbelfrakturen unter besonderer Berücksichtigung des Spongiosatransplantates und der morphologischen Bedingungen des Intervertebralraumes. Habilitationsschrift Fakultät für Klinische Medizin Mannheim 1998

\section{Dr. med. Franz Holz \\ Oberarzt}

Dr. med. Stefan Matschke

Chirurg, Unfallchirurg

Prof. Dr. med. Andreas Wentzensen

Ärztlicher Direktor

Berufsgenossenschaftliche Unfallklinik

Ludwig-Guttmann-Str. 13

D-67071 Ludwigshafen 